\title{
Rational use of medications: If Canada can't do it ...
}

\author{
Robert M. Califf MD
}

$\infty$ See related research paper by Jackevicius and colleagues, page E19

I $\mathrm{n}$ this issue, Jackevicius and colleagues offer a provocative evaluation of temporal changes in the use of cardiovascular drugs in Canada. ${ }^{1}$ By examining information from a national database managed by the prescriptiontracking giant IMS Health in conjunction with the Canadian government, these investigators reveal several findings that at first sight seem surprising in the light of Canada's discipline in the allocation of medical care. ${ }^{2}$ Jackevicius and colleagues show that the use of cardiovascular medications has grown steadily, that this growth is disproportionately evident in patent-protected medications and that variations exist between provinces. In addition, the increase displays an intriguing "east-to-west" gradient, with the largest increases seen in the easternmost provinces. The authors ominously conclude that these increases "may reach potentially unsustainable levels."

Information about prescribing is now available at a national level in many countries. It provides opportunities for exploring health care in ways that were impossible earlier. Although the Canadian prescription-tracking system examined in this study is far from perfect, it provides a summary of prescribing activity at the regional level that enables a critical analysis of policy with far-reaching implications. A much more rational system, in which reliable real-time information can be used to fine-tune prescribing, now seems within reach, given the evolution of local, regional and national electronic health records.

Three age-old questions of universal relevance arise from these data. First, why is there so much variation in medication use within a single country? Second, what is the right amount of medication use when the sum of costs, risks and benefits are weighed? Third, what can be done with this basic system (as well as those that will evolve in other countries) to take analysis of medication use to the next level of detail?

Variations in the use of health services have been shown within and among all countries in which this issue has been examined, regardless of the geographic unit of analysis. This observation has been most profoundly popularized in the Dartmouth Atlas of Healthcare (www.dartmouthatlas.org), based on the pioneering work of Jack Wennberg. ${ }^{3}$ The reasons for these variations are multifactorial and are currently a hot topic in health services research. Cultural norms among practitioners, reimbursement systems and individual preferences all play a part.

Ideally, therapeutic choices are made in the context of definitive clinical trials that show that a given decision will lead to improved health for the patient. Furthermore, when defini-

\section{Key points}

- Increasingly sophisticated medical and claims databases allow national patterns of medication use to be characterized at high levels of detail.

- Variations in these patterns of use raise questions about their underlying causes as well as their implications for health care.

- Continuing improvements in data resources may ultimately yield definitive answers to questions about the optimal level of medication use.

tive evidence about comparative choices and their relative costs exists, reasonable decisions about cost-effectiveness can be made. In the best of worlds, scientists funded by government agencies and private sources devise innovative technologies (drugs, devices, behavioural interventions), which are then developed through a disciplined series of evaluations, including preclinical development, early-phase studies and larger clinical trials, ultimately culminating in permission to market a therapy as safe and effective. ${ }^{4}$ This process progressively narrows the range of uncertainty regarding the benefits, risks and costs of using the technology. When this system works well, the result is "evidence-based medicine."

In reality, definitive evidence is often lacking, with many recommendations in clinical practice guidelines emanating from consensus among experts. Rigorous comparative effectiveness studies are in even shorter supply, and comparative effectiveness studies with robust cost-effectiveness analysis are rare. In the absence of the evidence that we need, we are left with opinions about whether the increase in the use of patented and expensive cardiovascular medications, described by Jackevicius and colleagues, is too much, just right or not enough.

The authors notably chose to focus on medications used to treat cardiovascular disease. Each drug class they evaluated has a proven use in reducing the risk of death and disability in patients with cardiovascular disease, or in patients who are at high risk of a cardiovascular event. In many other areas of medicine, this level of evidence is absent or is in short supply. Indeed, the sustained and dramatic reduction in death and disability from cardiovascular disease is partially due to access to these medications. ${ }^{5}$ Therefore, reasonable people might

Robert Califf is vice-chancellor for clinical research and director of the Duke Translational Medicine Institute and Donald F. Fortin is professor of cardiology, Duke University Medical Center, Durham, USA. 
agree with the "ominous" interpretation of the authors or could argue that this is good news because this uptake of proven medications is paralleled by a documented reduction in death and disability.

Determining the right level of medication use depends critically on the balance of total societal costs and health outcomes. This consideration is missing from the report by Jackevicius and colleagues. If greater use of cardiovascular medications controls blood pressure and lipid levels while also slowing progression of kidney disease and preventing adverse arterial and myocardial remodeling, then the cost of medications could easily be offset by a reduction in hospital admissions. Alternatively, the higher cost might result in a health benefit that would be considered well worth the incremental cost.

Even if a highly efficient system could be developed that would reduce the societal outlay for medications to the minimum level, the issue of impact on innovation will need to be addressed. So far the system of drug development has depended on the harrowing cycle described above, where the failure rate of molecular candidates is about $99.9 \%$. Even after introduction into human testing, less than $1 \%$ of molecules eventually see clinical use. ${ }^{6,7}$ If the use of new drugs were strictly limited, a different approach to providing capital for research and development would be needed to maintain a robust pipeline of innovative technologies. Switching to a system that rewards innovative drugs differentially would require both legal changes to patent laws and cultural changes in practice patterns.

Electronic medical records provide opportunities for benefit at multiple levels. The United States is embarking on the most expensive planned effort to automate medical records in history, with the allocation of US\$34 billion to the development and deployment of health information technology. Pharmaceutical use is only one facet of the far-reaching implications of this technology. However, universal electronic prescribing embedded in universal electronic health records could usher in a new era in which a model for continuous quality improvement would generate evidence at a greatly accelerated pace. The evidence would be incorporated into practice with much greater confidence that a given patient was receiving the best possible medication to prevent future death and disability.

Jackevicius and colleagues show us that accurate information can allow us to ask important questions. The next few years will offer a critical test of our collective will to develop better systems capable of answering those questions.

Competing interests: Robert Califf receives research grants from Merck, Novartis Pharmaceutical, Schering Plough Corporation and Scius. Payments for work performed under these contracts, portions of which support his salary, are made to Duke University. Dr. Califf also receives reimbursement for educational activities or consulting fees from Acumed, Amylin, the Annenberg Center, Avalere Health, Bayer, Biogen, Boehringer Ingelheim, Boston Scientific, Brandeis University, Five Prime, GlaxoSmithKline, Johnson \& Johnson, Heart.org (Conceptis), the Kowa Research Institute, Eli Lilly, Medtronic, Merck, Nitrox LLC, Novartis Pharmaceutical, Roche, Sanofi-Aventis, Schering Plough, Scius, Targacept, the University of Florida, Vertex and Vivus. Al revenues from educational activities are paid to Duke University; all personal income generated by consulting activities is donated to nonprofit organizations, with the majority being donated to the clinical research fellowship fund of the Duke Clinical Research Institute. Dr. Califf holds significant (> US\$10 000) equity in Nitrox LLC. A complete listing of financial disclosures for Dr. Califf from 2007-2009 is also available at http://dcri.org/research/coi.jsp.

\section{REFERENCES}

1. Jackevicius CA, Cox JL, Carreon D, et al. Long-term trends in use of and expenditures for cardiovascular medications in Canada. CMAJ 2009;181:45-51.

2. Mark DB, Naylor CD, Hlatky MA, et al. Use of medical resources and quality of life after acute myocardial infarction in Canada and the United States. $N$ Engl $J$ Med 1994;331:1130-5.

3. Wennberg JE. Unwarranted variations in healthcare delivery: implications for academic medical centres. BMJ 2002;325:961-4.

4. Califf RM, Peterson ED, Gibbons RJ, et al. Integrating quality into the cycle of therapeutic development. J Am Coll Cardiol 2002;40:1895-901.

5. Goldman L, Phillips KA, Coxson P, et al. The effect of risk factor reductions between 1981 and 1990 on coronary heart disease incidence, prevalence, mortality and cost. J Am Coll Cardiol 2001;38:1012-7.

6. DiMasi JA, Grabowski HG. The cost of biopharmaceutical R\&D: Is biotech different? Manage Decis Econ 2007;28:469-79.

7. Schachter AD, Ramoni MF. Clinical forecasting in drug development. Nat Rev Drug Discov 2007;6:107-8.

Correspondence to: Dr. Robert M. Califf, vice-chancellor for clinical research, Duke University Medical Center, Campus Box 3850, Durham NC 27710, USA; fax 919 668-7103; robert.califf@duke.edu 